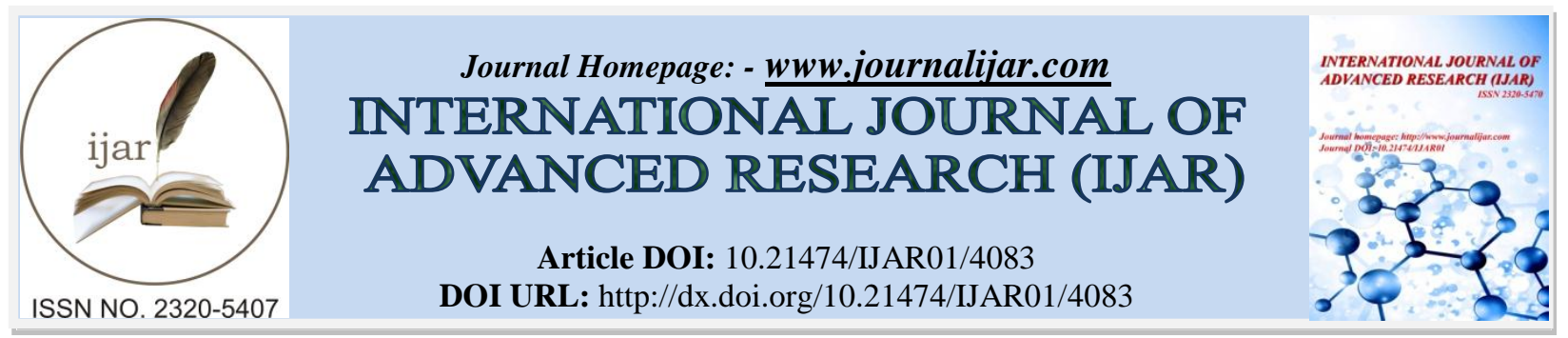

RESEARCH ARTICLE

\title{
MULTILEVEL IMAGE THRESHOLDING USING ARTIFICIAL INTELLIGENCE TECHNIQUES.
}

\author{
R. Sabeena ${ }^{1}$ and C. Subassree ${ }^{1}$. \\ Department of Electronics and Instrumentation Engineering, St. Joseph's College of Engineering, Chennai-600119.
}

\section{Manuscript Info}

(.........................

Manuscript History

Received: 05 March 2017

Final Accepted: 01 April 2017

Published: May 2017

Key words:-

Image Segmentation, Bilevel

thresholding, Multilevel thresholding,

Otsu-Tsallis method and MLTPSO.

\section{Abstract}

Analysis and comparison of various multilevel segmentation techniques to efficiently detect the tumourous region from MR Brain Images in early stages.To extract the abnormal tissues using multilevel image thresholding based on PSO. In the area of image processing, segmentation of an image into multiple regions is very important for classification and recognition steps. In this project we describe a novel method for segmentation of images based on Particle Swarm optimization for determining multilevel threshold for a given image. The proposed method is compared with other known multilevel segmentation methods to demonstrate its efficiency.

Copy Right, IJAR, 2017,. All rights reserved.

\section{Introduction:-}

Image segmentation is the processing technique used to regulate the features of an image. In imaging science, image processing plays a vital role in the analysis and interpretation of images in fields such as medical discipline, navigation, environment modeling, automatic event detection, surveillance, texture and pattern recognition, and damage detection. The development of digital imaging techniques and computing technology increased the potential of imaging science.

During the image processing operation, a photograph or a video frame is analyzed with a chosen signal processing technique and the outcomes such as processed image, data, and parameters related to image are further investigated to extract the desired information from the raw input image[1].

The goal of image segmentation is to partition a volumetric medical image into separate regions, usually anatomic structures (tissue types) that are meaningful for a specific task. It serves as a key in image analysis and pattern recognition and is a fundamental step toward low-level vision, which is significant for object recognition and tracking, image retrieval, face detection, and other computer-vision-related applications [2,3].

Image segmentation is considered as meaningful analysis and interpretation of image acquired. Usually this is regarded as a mandatory preprocessing step in many computer vision based applications, which is useful in extracting objects of interest from an acquired image by segregating them from rest of the scene[4].

Some of the different techniques used are,

Segmentation based on edge detection, Threshold method, Region based segmentation and Clustering based image segmentation. 


\section{Methodology:-}

Collection and segmentation of tumourous MR Brain Images by multilevel segmentation techniques. To increase the dimensionality the features are extracted. Compare and evaluate the performance of the proposed segmentation technique with` the classical multilevel and bilevel segmentation techniques.

\section{Database and Software:-}

The brain images were obtained from the open source website called med.harvard, Lung Image Database Consortium, MR Brain images with tumour for 4 patients, DICOM Images are converted to JPG format using radiant software. The software used is Matlab R2013a .

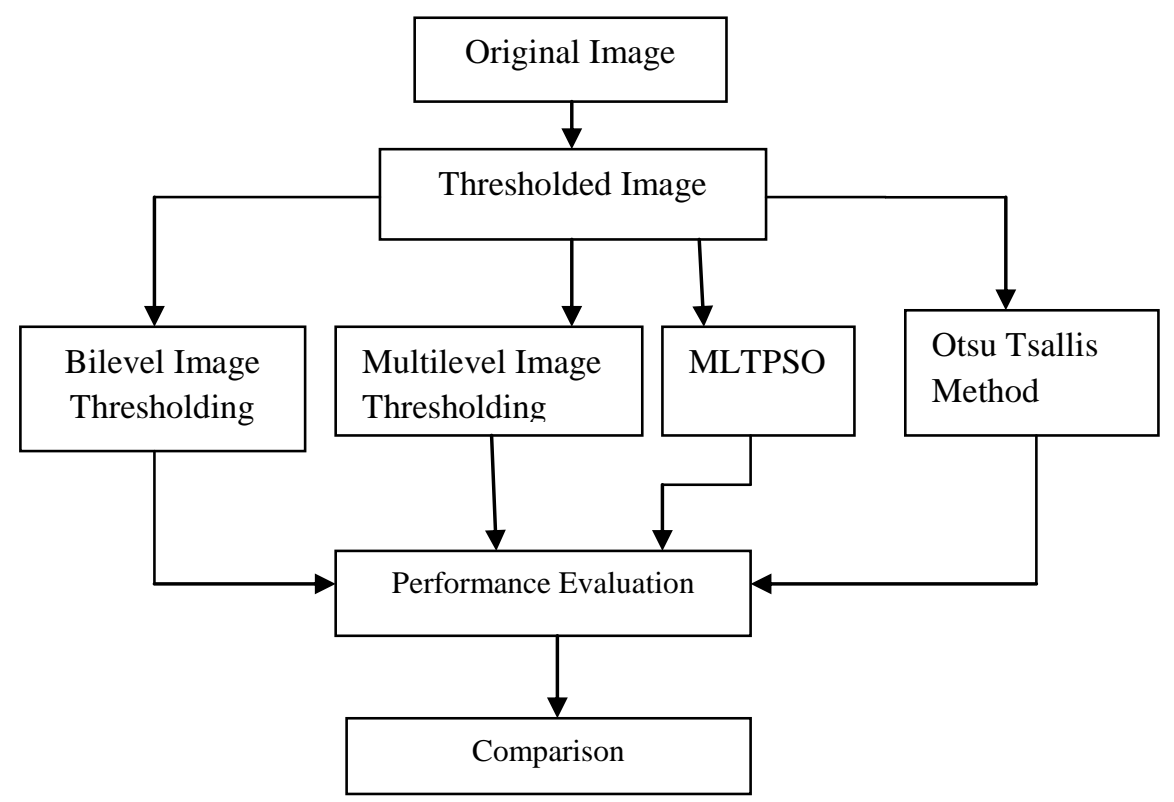

\section{B)Thresholding}

Fig. 1:- Workflow representation of Multilevel Image Thresholding

Thresholding is the methodology which is used to segment an image and isolate regions of interest from an image. Threshold values and number of class's parameters mainly affects the segmented output[5]. Sridevi.M et al has put forward a novel methodology utilizing evolutionary computing to select optimum threshold value.

The simplest method of image segmentation is called the thresholding method. This method is based on a clip-level (or a threshold value) to turn a gray-scale image into a binary image. There is also a balanced histogram thresholding. The key of this method is to select the threshold value (or values when multiple-levels are selected).

The simplest thresholding methods replace each pixel in an image with a black pixel if the image intensity is less than some fixed constant $T$ or a white pixel if the image intensity is greater than that constant[6].

Thresholding methods categorizes into the following groups based on the information the algorithm manipulates (Sezgin et al., 2004),

- Histogram shape based method

- Clustering based method

- $\quad$ Entropy based method

- Local method

There are two types of thresholding.

They are,

Bilevel Image Thresholding

Multilevel Image Thresholding

\section{B1) Bilevel Image Thresholding:-}

A binary image is a digital image that has only two possible values for each pixel. Binary images are also called $b i$ level or two-level .Typically, the two colors used for a binary image are black and white, though any two colors can 
be used. The color used for the object in the image is the foreground color while the rest of the image is the background color[1]. This means that each pixel is stored as single bit i.e., Oor1.Thenames black-and-white, $B \& W$, monochrome or monochromatic are often used for this concept, but may also designate any images that have only one sample per pixel, such as grayscale images. Binary images are produced from color images by segmentation.

\section{B2) Multilevel Image Thresholding:-}

A variety of multilevel thresholding approaches have been proposed for image segmentation including the Otsu criterion. Multilevel thresholding is a process that segments a gray level image into several distinct regions. This technique determines more than one threshold for the given image and segments the image into certain brightness regions, which correspond to one background and several objects. The major drawback of this problem is the computational effort that is much larger as the number of threshold levels increase[7].

The segmentation results are affected by factors such as number of threshold and threshold values. This proposes different methods for determining optimal thresholds using optimization techniques namely GA, PSO and hybrid model. Parallel algorithms are also proposed and implemented for these methods to reduce the execution time. Traditional methods work well for a bilevel thresholding problem, when the number of threshold level increases, complexity of the thresholding problem also will increase and the traditional method requires more computational time.

\section{C) Otsu Tsalli's Method:-}

Otsu's method, used to automatically perform clustering-based image thresholding. The methods such as Tsallis, and Otsu are widely adopted by most of the researchers to find solution for multilevel image segmentation problems[811]. In general, Otsu based thresholding techniques proved their better shape and uniformity measures for the bilevel and multilevel thresholding problems[3].

Consequently, Otsu's method is roughly a one-dimensional, Otsu's method is also directly related to the Jenks optimization method. The extension of the original method to multi-level thresholding is referred to as the multi Otsu method. Otsu is one of the commonly used methods in the segmentation of Gray scale and RGB image. Histogram Counts is a 256-element histogram of a grayscale image different gray-levels (typical for 8-bit images). Level is the threshold for the image (double).

It is one of the best global thresholding methods. Global thresholding uses only one threshold value, which is estimated based on statistics or heuristics on global image attributes, to classify image pixels into foreground or background.

\section{Proposed Work:-}

Optimisation Algorithm:-

Optimization algorithms are used before the segmentation of the image and after the feature extraction. Optimization algorithms are used to highlight the features that are necessary for reviewing. The optimization algorithms can be classified based on a number of parameters.

Some of the most recently developed algorithms are-

- Bacterial Foraging Optimization algorithm

- Particle Swarm Optimization algorithm

- Bat Optimization algorithm

- Glowworm Swarm Optimization algorithm

- Ant Colony Optimization algorithm

- Electro-Magnetism Optimization algorithm

- Bees Optimization algorithm

\section{MLTPSO:-}

PSO based approaches are widely considered in image segmentation application. In this work, MLTPSO algorithm is adopted to segment the image. Particle Swarm Optimization (PSO) is an algorithm inspired by bird-flocking and fish-schooling. The algorithm is population-based where a set of potential solutions evolve to approach an optimum solution for a problem. PSO method starts with random initialization of a population of particles in the search space and works on the social behaviour of the particles in the swarm[7]. The trajectory of each individual in the search 
space is adjusted by dynamically altering the velocity of each particle, according to its own flying experience and the flying experience of the swarm. It is a global optimization algorithm for dealing with problems in which a best solution can be represented as a point or surface in an n-dimensional space.

PSO is a computational method that optimizes a problem by iteratively trying to improve a candidate solution with regard to a given measure of quality. It solves a problem by having a population of candidate solutions, here dubbed as particles, and by moving these particles around in the search-space according to simple mathematical formulae over the particle's position and velocity. Each particle's movement is influenced by its local best known position but, is also guided toward the best known positions in the search-space, which are updated as better positions are found by other particles. This is expected to move the swarm toward the best solutions.

In this paper, a proposed algorithm for image segmentation based on PSO is used to automatically determinate the threshold values in multilevel thresholding problem. The PSO algorithm is based on swarm behavior of birds where particles (in our case: pixels), fly through the search space using two simples equations for velocity and position. The main advantage of such an approach over other global minimization strategies is that the large number of members that make up the particle swarm make the technique impressively resilient to the problem of local minima. It is very suitable to solve complex problem with multiple decision at low cost of computational time. As compared with other evolutionary computation algorithms, PSO has many advantages such as non-use of genetic operation; like crossover and mutation with Genetic Algorithm.

[7]PSO is an efficient algorithm that is based on a population initialized with a random solution called particle. Each particle represents an approximate solution to a complex problem in the search space. This solution is determined based on the collective experiences of the same swarm. In PSO, each particle is characterized by its own position vector and velocity vector.

The movement of these vectors in the search space is controlled by the following recursive equations:

$$
\begin{aligned}
& v_{i m}=w^{*} v_{i m}+c_{1} * \operatorname{rand} 1() *\left(p_{i m}-x_{i m}\right)+c_{2} * \operatorname{rand} 2() *\left(p_{g m}-x_{i m}\right) \ldots \ldots \ldots . .(1)
\end{aligned}
$$

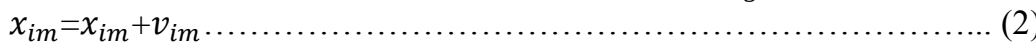

where $x_{i m}$ is the $i^{t h}$ position of the particle of the swarm; the velocity of this particle; $p_{i m}$ the best previous position of the $i^{t h}$ particle; $p_{g m}$ is the best position of particle in the swarm; $1 \leq m \leq M$ with $M$ is the search space; rand1() and rand2() are the rand2() are the two independents random number with uniform distribution in the range $[0,1] ; c_{1}$ and $c_{2}$ are two positives constants of accelerations coefficients called cognitive and social parameter respectively; $w$ is called inertia weight and it is used to control the balance between exploration and search space exploitation.

\section{Results and Discussions:-}

In this work the four algorithm such as Bilevel Thresholding, Multilevel Thresholding, MLT-Particle Swarm Optimisation(PSO) and Otsu Tsallis method are implemented using Matlab 2013a software under windows 10 operating system. The main aim of this work is to segment the tumorous tissues from the MR brain image with tumour. The four image segmentation algorithms have been used to segment the abnormal tissues. The results obtained on the application of these four algorithms have been shown in this chapter.

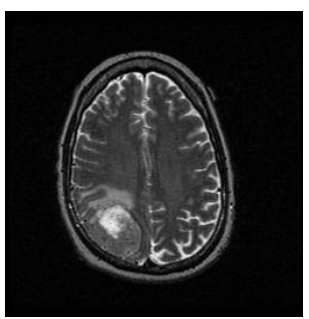

(a)

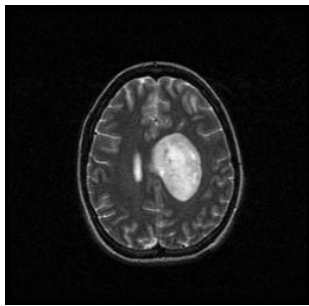

(b) 


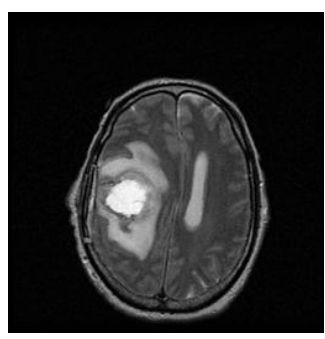

(c)

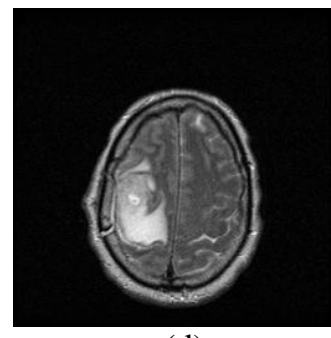

(d)

Fig. 2:- (a),(b),(c),(d)- MR Brain Input Images.

Implementaion of various techniques

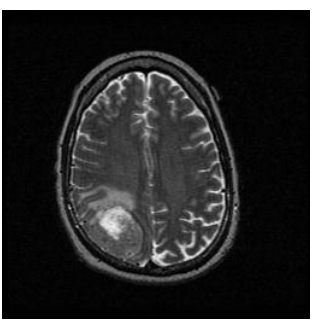

(a)

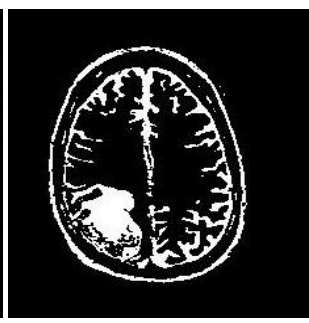

(b)

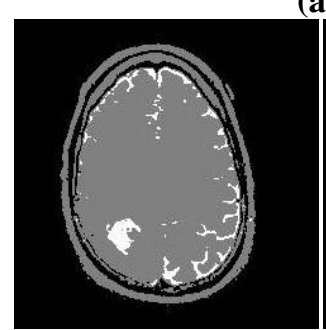

(c)

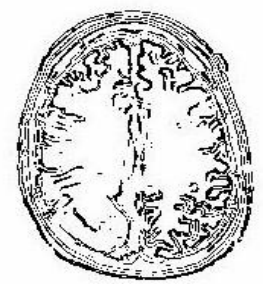

(f)

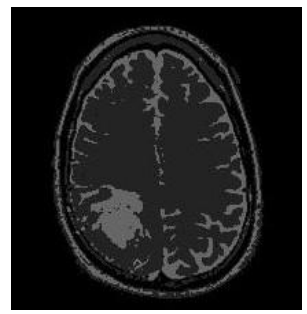

(i)

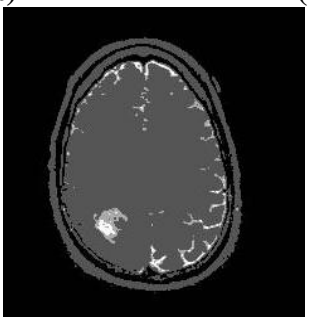

(d)

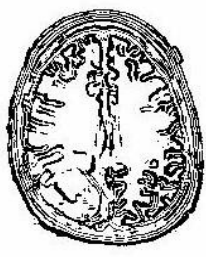

(g)

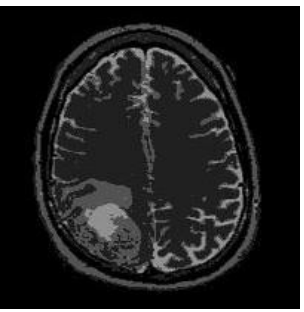

(j)

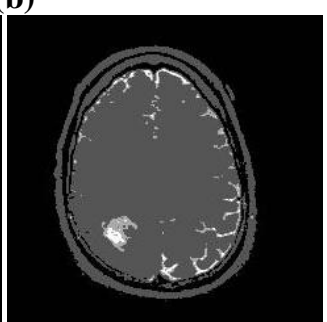

(e)

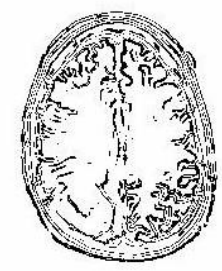

(h)

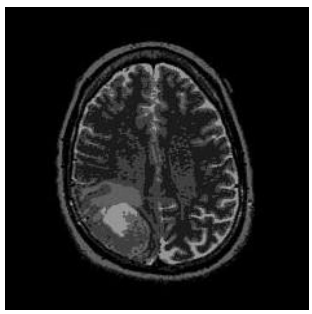

(k)

Fig. 3:- (a)-Original MR Image ; (b)-Bilevel thresholding for $n=2$; (c),(d),(e)-Multilevel thresholding for $n=3,4,5$; (f),(g),(h)- Otsu Tsallis method for $n=3,4,5 ;(\mathrm{i}),(\mathrm{j}),(\mathrm{k})$ - MLTPSO for $\mathrm{n}=3,4,5$.

\section{Performance Evaluation:-}

To evaluate the performance of the segmentation algorithm, there are many methods available. In this work, region non-uniformity between the neighbouring pixels, and computation time for each algorithm is compared. 
a) Region Non-Uniformity:-

This is the standard methods to evaluate the performance. This does not require ground truth information and is defined as,

$$
N U=\left[\left|F_{T}\right| /\left|F_{T}+B_{T}\right|\right]^{*}\left[\sigma_{t}^{2} / \sigma^{2}\right]
$$

where $\sigma^{2}$ represents the variance of the whole image, and $\sigma_{t}^{2}$ represents the foreground variance. $F_{T}$ and $B_{T}$ denotes the foreground and background area pixels in the test image. It is expected that a well segmented image will have a non-uniformity measure close to 0 , while the worst case corresponds to $\mathrm{NU}=1$.

b) Computation Time:-

The qualitative comparisons for the segmented results are shown in table below,

Table1:- For Bilevel Thresholding

\begin{tabular}{|l|l|}
\hline IMAGES & COMPUTED TIME (S) \\
\hline 1 & $0.451 \mathrm{~s}$ \\
\hline 2 & $0.803 \mathrm{~s}$ \\
\hline 3 & $0.268 \mathrm{~s}$ \\
\hline
\end{tabular}

Table2:- For Multilevel Thresholding

\begin{tabular}{|c|c|c|c|c|}
\hline IMAGES & PEAKS & RUN\& TIME & HISTOGRAM PEAKS & THRESHOLDS \\
\hline \multirow{3}{*}{1.} & P3 & $25.715 \mathrm{~s}$ & $\begin{array}{lll}8 & 56 & 160\end{array}$ & $34 \quad 156$ \\
\hline & $\mathrm{P} 4$ & $53.968 \mathrm{~s}$ & $\begin{array}{llll}8 & 56 & 160 & 216\end{array}$ & $34 \quad 156 \quad 209$ \\
\hline & P5 & $36.063 \mathrm{~s}$ & $4 \quad 56 \quad 160188216$ & $\begin{array}{llll}39 & 156 & 182 & 209\end{array}$ \\
\hline \multirow{3}{*}{2.} & P3 & $103.509 \mathrm{~s}$ & $\begin{array}{lll}8 & 64 & 160\end{array}$ & $41 \quad 152$ \\
\hline & $\mathrm{P} 4$ & $32.728 \mathrm{~s}$ & $\begin{array}{llll}8 & 64 & 128 & 160\end{array}$ & $41 \quad 109153$ \\
\hline & P5 & $25.903 \mathrm{~s}$ & $\begin{array}{lllll}8 & 64 & 128 & 160 & 240\end{array}$ & $\begin{array}{llll}41 & 109 & 153 & 198\end{array}$ \\
\hline \multirow{3}{*}{3.} & P3 & $18.978 \mathrm{~s}$ & $\begin{array}{lll}8 & 80 & 136\end{array}$ & $28 \quad 131$ \\
\hline & $\mathrm{P} 4$ & $22.354 \mathrm{~s}$ & $\begin{array}{llll}8 & 80 & 136 & 208\end{array}$ & $\begin{array}{lll}28 & 131 & 199\end{array}$ \\
\hline & P5 & $26.992 \mathrm{~s}$ & $\begin{array}{lllll}4 & 76 & 96 & 136 & 212\end{array}$ & $\begin{array}{llll}16 & 93 & 131 & 201\end{array}$ \\
\hline
\end{tabular}

Table3:- For MLTPSO Algorithm

\begin{tabular}{|c|c|c|c|c|c|c|}
\hline \multirow[t]{2}{*}{ IMAGES } & \multirow{2}{*}{$\begin{array}{l}\text { LEVEL } \\
\text { (n) }\end{array}$} & \multirow[t]{2}{*}{ RUN\& TIME } & \multicolumn{2}{|c|}{ INTENSITY } & \multirow[t]{2}{*}{ FITNESS } & \multirow{2}{*}{$\begin{array}{l}\text { TIME } \\
\text { (S) }\end{array}$} \\
\hline & & & Min & Max & & \\
\hline \multirow[t]{3}{*}{1.} & n3 & $7.048 \mathrm{~s}$ & 35 & 102 & 1.5582 & 6.1011 \\
\hline & $\mathrm{n} 4$ & $8.829 \mathrm{~s}$ & 32 & 144 & 1.6392 & 7.8690 \\
\hline & $\mathrm{n} 5$ & $10.687 \mathrm{~s}$ & 28 & 150 & 1.6630 & 9.6484 \\
\hline \multirow[t]{3}{*}{2.} & n3 & $6.921 \mathrm{~s}$ & 37 & 120 & 1.6238 & 6.1414 \\
\hline & $\mathrm{n} 4$ & $8.717 \mathrm{~s}$ & 32 & 145 & 1.6691 & 7.8748 \\
\hline & $\mathrm{n} 5$ & $10.602 \mathrm{~s}$ & 29 & 186 & 1.7204 & 9.6758 \\
\hline \multirow[t]{3}{*}{3.} & n3 & $6.828 \mathrm{~s}$ & 37 & 111 & 2.0992 & 6.0964 \\
\hline & $\mathrm{n} 4$ & $8.545 \mathrm{~s}$ & 36 & 184 & 2.1743 & 7.8126 \\
\hline & $\mathrm{n} 5$ & $10.303 \mathrm{~s}$ & 31 & 188 & 2.2147 & 9.5176 \\
\hline
\end{tabular}

Table4:- For Otsu Tsallis Method

\begin{tabular}{|l|l|l|l|}
\hline IMAGES & $\mathbf{n = 3}$ & $\mathbf{n = 4}$ & $\mathbf{n = 5}$ \\
\hline 1. & $17.667 \mathrm{~s}$ & $24.113 \mathrm{~s}$ & $18.127 \mathrm{~s}$ \\
\hline 2. & $24.043 \mathrm{~s}$ & $15.277 \mathrm{~s}$ & $14.294 \mathrm{~s}$ \\
\hline 3. & $94.606 \mathrm{~s}$ & $14.671 \mathrm{~s}$ & $21.041 \mathrm{~s}$ \\
\hline
\end{tabular}


Table5:- Performance Evaluation of Segmentation techniques

\begin{tabular}{|l|l|l|l|}
\hline $\begin{array}{l}\text { SEGMENTATION } \\
\text { TECHNIQUE }\end{array}$ & LEVELS & $\begin{array}{l}\text { REGION } \\
\text { NON-UNIFORMITY }\end{array}$ & COMPUTATION TIME \\
\hline BILEVEL & $\mathrm{n}=2$ & 0.2155 & $0.321 \mathrm{~s}$ \\
THRESHOLDING & & & $25.715 \mathrm{~s}$ \\
MULTILEVEL & $\mathrm{n}=3$ & 0.0176 & $53.968 \mathrm{~s}$ \\
THRESHOLDING & $\mathrm{n}=4$ & 0.0223 & $36.063 \mathrm{~s}$ \\
\hline MLTPSO & $\mathrm{n}=5$ & 0.0221 & $7.048 \mathrm{~s}$ \\
& $\mathrm{n}=3$ & 0.2604 & $8.829 \mathrm{~s}$ \\
& $\mathrm{n}=4$ & 0.2597 & $10.687 \mathrm{~s}$ \\
\hline OTSU & $\mathrm{n}=5$ & 0.2600 & $26.636 \mathrm{~s}$ \\
+TSALLI'S & $\mathrm{n}=3$ & 0.0028 & $13.518 \mathrm{~s}$ \\
& $\mathrm{n}=4$ & 0.0126 & $15.483 \mathrm{~s}$ \\
\hline
\end{tabular}

Thus, from the above table it is inferred that values closer to zero in region non-uniformity produces good segmentation results. The variation in values is due to variation in gray levels of the pixels. Hence, the proposed method is preferred as inferred from the table.

\section{Conclusion:-}

From the experimental results, it is inferred that proposed method MLTPSO takes less computation time when compared with the other multilevel thresholding techniques. The optimal threshold can be obtained easily with high efficiency. From the above table, we infer that the huge computation time involved in the other multilevel techniques is reduced to a greater extent in the proposed method. Thus, reducing the total time involved in automated image diagnosis. The performance evaluation of proposed segmentation algorithm proves its efficiency. In this, MLTPSO inspired Particle Swarm Optimization algorithm for multilevel thresholding is developed. This method is able to determine optimal threshold values from complex gray-level images. Experimental results demonstrated by computing optimal threshold values in 3 different levels (3, 4, and 5 levels) is shown. Moreover, due to the low computational complexity of the algorithm, this algorithm will be applied to classify other MR medical images.

\section{References:-}

1. N.SriMadhavaRaja, V.Rajinikanth, and K.Latha "Otsu Based Optimal Multilevel Image Thresholding Using Firefly Algorithm" Modelling and Simulation in Engineering, Hindawi,vol 2014.

2. N. Pal, and S. Pal, “A review on image segmentation techniques, Pattern Recognition”, Vol. 26, No. 9,1993, pp. $1277-1294$.

3. P. D. Sathya and R. Kayalvizhi, "Modified bacterial foraging algorithm based multilevel thresholding for image

4. segmentation" Engineering Applications of Artificial Intelligence, vol. 24, no. 4, pp. 595-615, 2011.

5. Madhubanti Maitra, Amitava Chatterjee, "A hybrid cooperative-comprehensive learning based PSO algorithm for image segmentation using multilevel thresholding".

6. T.Abimala,S.Gayathri, “A Literature Review On Heuristic Algorithms In Image Segmentation Applications”, International Journal Of Pharmacy \& Technology,March-2016, vol. 8, Issue No.1,10535-1054.

7. Zhang, Y. (2011). "Optimal multi-level Thresholding based on Maximum Tsallis Entropy via an Artificial Bee Colony Approach", Entropy.13.

8. Fayçal HAMDAOUI, Anis SAKLY, Abdellatif MTIBAA, "An efficient multithresholding method for image segmentation based on PSO".

9. P. D.Sathya and R. Kayalvizhi, "Optimum multilevel image thresholding based on Tsallis Eetropy method with bacterial foraging algorithm,” International Journal of Computer Science Issues, vol. 7, no. 5, pp. 336-343, 2010.

10. S. P. Duraisamy and R. Kayalvizhi, "A new multilevel thresholding method using swarm intelligence algorithm for image segmentation," Journal of Intelligent Learning Systems and Applications, vol. 2, pp. 126-138, 2010.

11. S. Sarkar, S. Das, and S. S. Chaudhuri, "Multilevel image thresholding based on Tsallis entropy and differential evolution," in Proceedings of the 3rd International Conference on Swarm,Evolutionary, and Memetic Computing (SEMCCO '12), B.K.Panigrahi, S.Das,

12. P.N. Suganthan, and P. K. Nanda, Eds., vol.7677 of Lecture Notes in Computer Science, pp. 17-24, Springer,2012.

13. K. Manikantan, B. V. Arun, and D. K. S. Yaradoni, "Optimal multilevel thresholds based on Tsallis entropy method using golden ratio particle swarm optimization for improved image segmentation,” Procedia Engineering, vol. 30, pp. 364-371, 2012. 